\title{
Asociación entre el autoconcepto académico con el rendimiento académico en estudiantes universitarios físicamente activos e inactivos Association between academic self-concept with academic achievement in physically active and inactive university students
}

Relmu Gedda Muñoz, Pablo Valdés Badilla, NataliaVillagrán del Picó Universidad Autónoma de Chile (Chile)

\begin{abstract}
Resumen. La presente investigación tiene por objetivo asociar el autoconcepto académico con el rendimiento académico en estudiantes universitarios chilenos físicamente activos e inactivos. Estudio transversal que evaluó a 1383 estudiantes de una universidad privada, utilizando la escala de autoconcepto académico (EAA), el registro de notas para obtener el rendimiento académico y la práctica de actividad física por medio de una pregunta dicotómica. Se realizaron correlaciones bivariadas entre laEAA, dimensión de autoeficaciaacadémica (AEA) y dimensión derendimiento percibido (RP) con el rendimiento académico a través de la correlación de Spearman y asociaciones por medio de Chi-Cuadrado de Pearson, diferenciando los resultados obtenidos entre el total de la muestra, los estudiantes físicamente activos (EFA) y estudiantes físicamente inactivos (EFI). LOS principales resultados indican correlación directay asociación significativa ( $p$ valor $<.01$ ) entrelaEAA y sus dimensionesAEA y RP con el rendimiento académico en el total de la muestra, mientras que al diferenciar en EFA y EFI, ambos grupos presentan correl ación y asociación entre laEAA y el rendimiento académico, lo quemanifiestaquelaprácticade actividad física no resultaser una variable que al tere los resultados de la muestratotal. En conclusión, a mayor autoconcepto académico mejor es el rendimiento académico en estudiantes universitarios, independientementea la práctica de actividad física.
\end{abstract}

Palabras claves: Autoconcepto, éxito académico, actividad física, estudiantes, pregrado.

\begin{abstract}
The aim of this research is to associate academic self-concept with academic achievement of physically active and inactive Chilean university students. The study is cross-sectional and eval uated 1383 students from a private university, using the academic self-concept scale (ASCS), the record of grades to obtain academic performance and a dichotomous question about physical activity practice. Bivariate correlations were made between theASCS, academic self-efficacy dimension (ASE) and perceived performance dimension (PP) with the academic achievement through Spear man's correlation and associations through Pearson's Chi-Square, differentiating the results obtained between the total sample, physically active students(PHAS) and physically inactive students (PHIS). The main results indicate a direct correlation and significant association ( $p$ val ue $<.01$ ) between ASCS and itsASE and PP dimensions with academic performance in the total sample, while when differentiating on PHAS and PHIS, both groups show correlation and association between ASC S and academic performance; this shows that the practice of physical activity does not turn out to be a variable that alters the results of the total sample. In conclusion, when students present a greater academic self-concept it is evident that they have better academic performance, regardless of the practice of physical activity.
\end{abstract}

Key words: Self-concept; academic success; physical activity; students; undergraduate.

\section{Introducción}

Los sistemas de educación superior han sufrido múltiples cambios a nivel global, influenciados mayormente por la ampliación de la cobertura y matrícula, avances tecnológicos y las demandas sociales que movilizan permanentes ajustes a sus estructuras y formas de operar (Altbach, Reisberg \& Rumbley, 2009; Bernasconi, 2015; Brunner \& Ganga, 2016; Ferreyra, Avitabile, Botero-Álvarez, Haimovich \& U rzúa, 2017). En Chile,

Fecha recepción: 05-08-20. Fecha de aceptación: 23-11-20

Relmu Antonio Gedda M uñoz

relmu.gedda@uautonoma.cl la educación superior ha sido protagonista del mismo fenómeno, debido ala creación de nuevas instituciones, carreras y programas de estudio (Teixeira, Sunwoong, Landoni \& Zulfiqar, 2017), el aumento de modalidades y prestaciones financieras para los estudiantes (Gazzola \& Didriksson, 2008; Espinoza \& González, 2016) y la diversificación de los perfiles de ingreso, hechos que en su conjunto, configuran una serie de desafíos para las universidades(Araneda-Guirriman, Sallán, Pedraja-Rejas \& Rodríguez-Ponce, 2018).

Con la ampliación de la cobertura y acceso a la educación superior, han surgido otros fenómenos asociados que se han vuelto necesarios de evaluar, como la comprensión de la experiencia de ingreso y permanencia en la educación superior (Donoso, Donoso \& Arias, 2010; 
Gedda, 2016), a través del conocimiento de los perfiles psicoeducativos de sus estudiantes, con el fin de promover acciones que permitan apoyar sus trayectorias educativas y académicas. En este entendido, la importancia de las variables socioafectivas (D onoso \& Schiefel bein, 2007) como parte de las características individuales del estudiante, relacionadas con la continuidad de estudios (Rodríguez, Fita \& Torrado, 2004; Carvajal, González $\&$ Sarzoza, 2018), son de alta relevancia para comprender las tasas de éxito, de rendimiento académico y de permanencia. U na de las variables socioafectivas más relevantes es el autoconcepto (Shavelson, Hubner \& Stanton, 1976), el cual ha sido estudiado en el contexto académico en diversas investigaciones ( $M$ arsh \& Shavelson, 1985; Hattie, 1992; M arsh \& Craven, 1997; Cardenal \& Fierro, 2003; M arsh \& Hau, 2003; Ferla, Valcke \& Cai, 2009; M arsh \& M artin, 2011; Khalaila, 2015; M éndez \& Gálvez, 2018). En relación a ello, el autoconcepto académico corresponde a un conjunto de percepciones, sentimientos, imágenes, atribuciones y juicios de valor referidos a sí mismo (Schmidt, Messoulam \& Molina, 2008), siendo estos juicios tanto descriptivos como evaluativos, expresando el modo en que la persona se representa, conoce y valora a ella misma, impactando directamente en las formas como enfrentan diversas situaciones (Garaigordobil \& Durá, 2006). En consecuencia, el autoconcepto académico es definido como la configuración organizada de percepciones relativamente estables que reflejan una evaluación de comportamientos y atributos académicos, y corresponde a un elemento socioafectivo que involucra la autoevaluación de la confianza personal respecto de las habilidades propias para resolver tareas, así como la efectividad percibida respecto a las propias competencias para el ámbito académico (Schmidt, M essoulam \& Molina, 2008).

Del mismo modo, el rendimiento académico constituye una variable ineludible cuando se trata de anal izar realidades universitarias, y se entiende como el resultado de diversos factores que interactúan entre sí, tales como: aspectos personales, relacionales, sociales, institucionales, experiencias educacionales, expectativas previas, entre otros (Díaz, A podaca-U rquijo, Arias, Escudero, Rodríguez \& Vidal, 2002). Como involucra diversos factores, es conveniente abordarlo desde más de una dimensión, o bien, desde los resultados de la combinación de esos factores, lo que se consigue normalmente mediante las notas obtenidas y la aprobación de cursos en un período académico dado (Garbanzo, 2007).
Por otra parte, se ha reportado una relación positiva entre el autoconcepto con mayor actividad física en estudiantes universitarios ( 0 netti- 0 netti, C hinchillaMinguet, Martins \& Castillo-Rodriguez, 2019), situación relevante debido a que la práctica regular de actividad física puede conducir a mejoras en la autoestima de adolescentes a corto plazo (Biddle \& A sare, 2011) y beneficios sobre la memoria, proceso de síntesis y degradación de los neurotransmisores, debido al aumento en el transporte de oxígeno al cerebro (Grissom, 2005; Rudolph \& McAuley, 1995; Ruiz-Hermosa, AlvarezBueno, Cavero-Redondo, Martinez-Vizcaino, Redondo-Tébar \& Sánchez-López, 2019). Además, poseer una buena condición física se ha asociado con mayor rendimiento académico en estudiantes universitarios (GodoyCumillaf, et al. , 2015; Iglesias, Planells\& M olina-López, 2019; Ruiz-Ariza, De la Torre-Cruz, Suárez-M anzano \& Martínez-López, 2018; Santana, Azevedo, Cattuzzo, Hill \& Andrade, 2017), por lo tanto, la práctica de actividad física de los estudiantes se ha vuelto esencial para favorecer el buen desempeño académico. En suma a lo anterior y considerando los nuevos escenarios el sistema de educación superior y los nuevos desafíos para las institucionesy programas, la presente investigación tiene por objetivo asociar el autoconcepto académico con el rendimiento académico en estudiantes universitarios chilenos físicamente activos e inactivos.

\section{Material y método}

Se realizó un estudio cuantitativo, descriptivo y correlacional, de corte transversal (Latorre, Arnal \& Del Rincón, 2003).

\section{Participantes}

La muestra fue seleccionada bajo un criterio no probabilístico accidental y estuvo constituida por 1383 estudiantes ( $74.1 \%$ mujeres y $25.9 \%$ hombres), pertenecientes a las siete facultades (administración y negocios; arquitectura y construcción; ciencias sociales; derecho; educación; ingeniería y salud) de una U niversidad privada de Chile. La edad de los encuestados presentó una media de 22.3 años. Los criterios de inclusión fueron: a) poseer 18 años o más; b) ser estudiante regular y con matrícula vigente en la universidad anal izada. Los criterios de exclusión fueron: a) estudiantes en proceso de titulación; b) estudiantes que respondieron parcialmente el instrumento.

Todos los participantes fueron informados de los alcances y propósito del estudio mediante un consenti- 
miento informado que autoriza el uso de la información con fines científicos. El protocolo de investigación fue desarrollado siguiendo lo expuesto en la Declaración de Helsinki (Velasquez, 2013).

\section{Autoconcepto académico}

Se utilizó la escalade autoconcepto académico (EAA) de Schmidt, Messoulam \& Molina (2008), adaptada y validada para el contexto universitario chileno por Méndez \& Gálvez (2018), reportando valores alfa de Cronbach y omega de $\mathrm{McD}$ onald de á $=.802$ y ù $=$ .867 parala dimensión de autoeficaciaacadémica(AEA); y de á $=.753$ y ù $=.787$ para la dimensión de rendimiento percibido (RP). El instrumento corresponde a una escala autoadministrable que evalúa el constructo del autoconcepto académico mediante dos dimensiones correlacionadas (AEA y RP).

El instrumento está compuesto por 12 ítems, con una valoración que va de 1 a 5 puntos presentadas a través de una escala tipo Likert con categorías posibles de respuesta desde «totalmente de acuerdo» hasta «totalmente en desacuerdo». Los ítems 1, 2, 3, 5, 10 y 11 se presentan con una redacción positiva (a mayor valor mejor percepción), mientras que los ítems 4, 6, 7, 8, 9 y 12 presentan una redacción negativa (a menor valor mejor percepción). La dimensión AEA cuenta con 7 ítems (1, 2, 3, 4, 5, 10 y 11) y un puntaje máximo de 35 puntos, mientrasqueladimensión RP cuentacon 5 ítems $(6,7,8,9$ y 12) con un puntaje máximo de 25 puntos. Los valores totales para la EAA varían entre 12 y 60 puntos, y tanto la EAA como sus dimensiones se interpretan considerando que, a mayor puntaje, mejor es el autoconcepto académico, AEA y RP de los estudiantes.

Por otra parte, para la presente investigación, se estructuraron cinco categorías para interpretar los resultados de la EAA y sus dimensiones (AEA y RP). De este modo, se establecieron puntos de corte en base a los puntajes mínimos y máximos obtenidos en cada dimensión y en la escala total, vinculando esos puntos de corte a categorías cualitativas. Para la EAA se consigna ron las siguientes categorías: baja (12 a 21 puntos); mediabaja (22 a 31 puntos); media (32 a 41 puntos), mediazalta (42 a 51 puntos) y alta (sobre 51 puntos). Parael caso de la dimensión AEA se estableció: baja (7 a 12 puntos); mediarbaja (13 a 18 puntos); media (19 a 24 puntos), media-alta (25 a 30 puntos) y alta (sobre 30 puntos). Y para el caso de la dimensión RP: baja (5 a 9 puntos); media-baja (10 a 13 puntos); media (14 a 17 puntos), media-alta (18 a 21 puntos) y alta (sobre 21 puntos).

\section{Rendimiento académico}

Para medir el rendimiento académico se consultaron los registros de desempeño académico de los estudiantes pertenecientes a las cohortes situadas entre 2012 y 2018. De este modo, se obtuvieron dos variables principales: las notas de cada estudiante en el total de asignaturas del año académico 2018; y el número de asignaturas inscritas, tanto las aprobadas como las reprobadas para el primer y segundo semestre del año académico 2018. Las notas obtenidas por un estudiante son entendidas como los promedios del total de notas de las asignaturas cursadas en el semestre académico, mientras que los porcentajes de aprobación de asignaturas corresponden a la proporción de las asignaturas aprobadas respecto al total de las asignaturas inscritas, resultando ambos en indicadores precisos y accesibles para valorar el rendimiento (Vélez \& Roa, 2005; Véliz-Burgos, Dörner \& Sandoval, 2016), dado que son un reflejo de los logros académicos en los diferentes componentes del aprendizaje incluyendo aspectos personales, académicos, sociales y propios de la formación profesional (Rodríguez, Fita \& Torrado, 2004).

\section{Práctica de actividad física}

Esta variable se obtuvo mediante una pregunta dicotómica aplicada a la totalidad de estudiantes evaluados: ¿haces más de 150 minutos de actividad física moderada 075 minutos de actividad física vigorosa a la semana?, la cual tenía como respuesta dos posibilidades: sí o no, lo que permitió clasificar a los estudiantes como físicamente activos (EFA) o estudiantes físicamente inactivos (EFI). Dicha pregunta se basó en los parámetros establecidos por el Colegio Americano de M edicina del Deporte (Garber, 2011) para considerar a una persona como físicamente activa y fue incorporada en la encuesta sobre autoconcepto académico.

\section{Procedimiento}

El proceso de aplicación de la EAA y la pregunta sobre práctica de actividad física fue mediante un cuestionario digital en línea de carácter voluntario y autoadministrado, donde los encuestados debían leer el objetivo del estudio, los criterios de utilización y manejo de los datos recolectados, así como aceptar explícita mente un consentimiento informado. Los cuestionarios fueron remitidos individualmente, por tanto, se respondieron consul tas o comentarios de los encuestadosy también de aquellos estudiantes que no aceptaron participar del estudio. Por su parte, el rendimiento académico fue obtenido desde las bases de datos del registro 
académico universitario. A partir de estos datos se calcularon los promedios anual es de notas y los porcenta jes de aprobación de asignaturas. Posteriormente se obtuvieron los valores media de las notas, del porcenta je de aprobación y de la EAA y sus dimensiones para generar variables categóricas del rendimiento académico y del autoconcepto académico.

\section{Análisis de datos}

Se utilizó el programa SPSS (Statistical Package for the Social Sciences) versión 25.0. Las variables fueron sometidas ala pruebade normalidad de Kolmogorov-Smirnov y a un análisis descriptivo cal culando frecuencias, distribuciones, media aritmética, mediana, desviación estándar, mínimo, máximo y porcentajes según tipo de variable. Se realizaron correlaciones bivariadas entre la EAA con el rendimiento académico a través de la prueba de correlación de Spearman, ya que los datos presentaron un comportamiento no normal. Adicionalmente, se recodificó la EAA y las variables de rendimiento académico, tanto promedio anual de notas como porcentaje de aprobación de asignaturas sobre el total de asignaturas inscritas, en variables categóricas ( $0=$ bajo la media y $1=$ sobre la media) para asociarlas a través de Chi-Cuadrado de Pearson. Para realizar los análisis descritos, la muestra fue distribuida en EFA, EFI y total de la muestra. Para todos los casos se consideró un nivel de significancia de $p<.05$.

\section{Resultados}

LaTabla 1 presenta los puntajes para la EAA y sus dimensiones, así como los estadísticos descriptivos sobre el rendimiento académico de los estudiantes evaluados. Se aprecia que los estudiantes evaluados al canzan una media de 48.8 puntos en laEAA, situándolos en la categoría «media-alta». La dimensión de AEA logró una media de 30.4 puntos, clasificando a los estudiantes en la categoría «alta». Mientras que la dimensión de RP consiguió una media de 18.4 puntos ubicándolos en la categoría «mediazalta». Por su parte, se advierte que los estudiantes aprueban, en promedio, un 93.2\% de su carga académica anual y que el promedio anual de notas es de 5.31 en una escala de 1 a 7. El 70.8\% de los estudiantes se encuentra sobre la media de aprobación de asignaturas y el $52.7 \%$ está sobre la media del promedio anual de notas.

En cuanto a los resultados sobre práctica de actividad física, en laTabla 2 se puede apreciar que el $36 \%$ de los estudiantes se declara físicamente activo y el $64 \%$ como físicamente inactivo. Además, los hombres son más activos (54\%) que las mujeres (29.7\%).

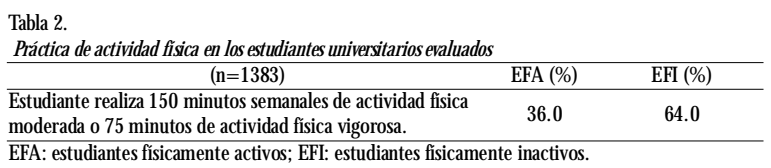

En laTabla 3 se aprecia que existe correlación positiva y significativa $(p<.01)$ entre el total de la EAA, dimensión de AEA y dimensión RP con el porcentaje de aprobación de asignaturas y el promedio anual de notas en el total de la muestra, mientras que los mismos resultados se aprecian al diferenciar la muestra entre EFA y EFI, aunque la fuerza estadística presenta variaciones entre ambos grupos, correlacionando con mayor fuerzala EAA y dimensión RP con losEFA cuando se trata del promedio anual de notas, mientras la dimensión AEA correlaciona con mayor fuerza en el grupo EFI, tanto mediante el porcentaje de aprobación de asignaturas, como mediante el promedio de anual de notas. No se reportaron diferencias significativas entre las variables al distribuir la muestra por sexo.

Tabla 1.

Autoconcepto académico y rendimiento académico de los estudiantes universitarios eval uados

\begin{tabular}{|c|c|c|c|c|c|c|c|c|c|}
\hline \multirow{2}{*}{$(n=1383)$} & & \multirow{2}{*}{ Puntaje M edia } & \multirow{2}{*}{ Mediana } & \multirow{2}{*}{ DE } & \multicolumn{5}{|c|}{ Escala (\%) } \\
\hline & & & & & 1 & 2 & 3 & 4 & 5 \\
\hline & 1. Soy capaz de hacer bien los trabajos y tareas, aunque sean dificiles. & 4.3 & 4.0 & 0.8 & 2.0 & 0.9 & 8.1 & 42.4 & 46.6 \\
\hline & 2. Si me esforzara lo suficiente, podría aprobar las asignaturas de este semestre. & 4.7 & 5.0 & 0.8 & 2.5 & 0.8 & 3.1 & 15.5 & 78.1 \\
\hline & 3. Creo que podré obtener buenas notas este semestre. & 4.5 & 5.0 & 0.8 & 2.2 & 1.0 & 6.1 & 31.3 & 59.4 \\
\hline Ítems dimensión AEA & 4. Aunque me effuerce, siempre me va a ir mal en la universidad. & 4.4 & 5.0 & 0.9 & 1.4 & 3.9 & 8.8 & 28.0 & 57.8 \\
\hline & 5. Soy capaz de mantener un buen rendimiento a lo largo de todo un semestre. & 4.0 & 4.0 & 0.9 & 1.9 & 4.4 & 19.8 & 40.5 & 33.4 \\
\hline & 10. Soy capaz de realizar buenos trabajos en clase. & 4.2 & 4.0 & 0.9 & 1.9 & 2.7 & 12.9 & 41.6 & 40.9 \\
\hline & 11. Si me dedico a fondo, puedo estudiar cualquier materia. & 4.5 & 5.0 & 0.9 & 2.6 & 1.6 & 5.7 & 23.4 & 66.7 \\
\hline \multirow[t]{3}{*}{ Total dimensión AEA } & Resultado total de ítems dimensión & 30.4 & 31.0 & 4.6 & 2.1 & 2.2 & 9.2 & 31.8 & 54.7 \\
\hline & 6. Soy lento para aprender. & 3.7 & 4.0 & 1.1 & 3.6 & 13.2 & 20.3 & 32.4 & 30.4 \\
\hline & 7. Cometo muchos errores cuando hago trabajos y tareas de la universidad. & 3.7 & 4.0 & 1.0 & 1.7 & 11.7 & 23.2 & 42.5 & 20.9 \\
\hline \multirow[t]{3}{*}{ Ítems dimensión RP } & 8. Me olvido fácilmente de lo que aprendo. & 3.6 & 4.0 & 1.0 & 3.3 & 10.8 & 29.1 & 35.1 & 21.7 \\
\hline & 9. Me cuesta entender lo que leo. & 3.9 & 4.0 & 1.0 & 2.8 & 8.5 & 19.6 & 38.3 & 30.8 \\
\hline & 12. Me resul ta difícil estudiar. & 3.5 & 4.0 & 1.2 & 6.1 & 13.7 & 26.2 & 30.2 & 23.9 \\
\hline Total dimensión RP & Resultado total de ítems dimensión & 18.4 & 19.0 & 3.8 & 3.5 & 11.6 & 23.7 & 25.5 & 35.7 \\
\hline \multirow[t]{2}{*}{ Total EAA } & Resultado total EAA & 48.8 & 49.0 & 7.0 & 11.9 & 16.2 & 15.2 & 23.4 & 33.4 \\
\hline & & \multicolumn{4}{|c|}{ Sobre el promedio } & \multicolumn{4}{|c|}{ Bajo el promedio } \\
\hline \multirow{2}{*}{\multicolumn{2}{|c|}{ Variable categorizada de EAA }} & \multicolumn{3}{|c|}{$57,0 \%$} & & \multicolumn{4}{|c|}{$43,0 \%$} \\
\hline & & & & Mediana & \multirow{2}{*}{\multicolumn{2}{|c|}{14.2}} & \multicolumn{2}{|c|}{ SM (\%) } & BM (\%) \\
\hline \multirow{2}{*}{\multicolumn{2}{|c|}{ Rendimiento académico }} & \multicolumn{2}{|c|}{93.2} & 100 & & & \multirow{2}{*}{\multicolumn{2}{|c|}{$\begin{array}{l}10.0 \\
52.7\end{array}$}} & 29.2 \\
\hline & & \multicolumn{2}{|l|}{5.31} & 5.4 & \multicolumn{2}{|c|}{$\begin{array}{c}14.2 \\
0.7\end{array}$} & & & 47.3 \\
\hline
\end{tabular}

acuerdo; 4 De acuerdo; 5 Totalmente de acuerdo. SM: estudiantes sobre la media; BM: estudiantes bajo la media 
Correlación entre la escala de autoconcepto académico con el rendimiento académico en estudiantes universitarios físicamente acti vos e inactivos

\begin{tabular}{|c|c|c|c|c|c|c|}
\hline & \multicolumn{6}{|c|}{ EAA } \\
\hline & \multicolumn{2}{|c|}{ MT } & \multicolumn{2}{|c|}{ EFA } & \multicolumn{2}{|c|}{ EFI } \\
\hline & Valor $r$ & Valor $p$ & Valor $r$ & Valor $p$ & Valor $r$ & Valor $p$ \\
\hline $\begin{array}{l}\text { Porcentaje de Aprobación } \\
\text { Asignaturas }\end{array}$ & .248 & .000 & .209 & .000 & .273 & .000 \\
\hline Promedio anual de notas & .304 & .000 & $\begin{array}{c}.309 \\
\text { D-AEA }\end{array}$ & .000 & .305 & .000 \\
\hline $\begin{array}{l}\text { Porcentaje de Aprobación } \\
\text { Asignaturas }\end{array}$ & .291 & .000 & .248 & .000 & .320 & .000 \\
\hline Promedio anual de notas & .341 & .000 & $\begin{array}{r}.314 \\
\text { D-RP }\end{array}$ & .000 & .361 & .000 \\
\hline $\begin{array}{l}\text { Porcentaje de Aprobación } \\
\text { Asignaturas }\end{array}$ & .129 & .000 & .120 & .000 & .139 & .000 \\
\hline Promedio anual de notas & .180 & .000 & 228 & .000 & 157 & .000 \\
\hline
\end{tabular}

EAA: escala autoconcepto académico; MT: muestra total; EFA: estudiantes físicamente activos: EFl: estudiantes físicamente inactivos: D-AEA: dimensión autoeficacia académica. D-RP. dimensión rendimiento percibido. Val or $r$ : fuerza estadística; valor $p$ : significancia estadística, utilizando la correlación de Spearman.

En laTabla 4 se advierte asociación estadísticamente significativa $(p<.01)$ entre el total de la EAA, dimensión de AEA, dimensión de RP con el porcentaje de aprobación de asignaturas y el promedio anual de notas para el total de la muestra. Por otra parte, al diferenciar la muestra, los EFA presentan una asociación estadísticamente significativa $(p<.01)$ entre el total de la EAA y la dimensión AEA con el rendimiento aca démico, siendo más intensas las asociaciones del promedio anual de notas que el porcentaje de aprobación de asignaturas. En cuanto a la dimensión RP, el porcentaje de aprobación de asignaturas no presenta asociación estadísticamente significativa con los EFA, mientras el promedio anual de notas sí tiene una asociación significativa ( $p$ <.001). Por su parte, los EFI presentan asocia ciones estadísticamente significativas $(p<.05)$ entre la EAA, dimensión AEA y dimensión RP con el rendimiento académico, no obstante esta última dimensión posee una asociación de baja intensidad. Al diferenciar la muestra por sexo no se reporta asociación.

Tabla 4

Asociación entre la escala de autoconcepto académico con el rendimiento académico en estudiantes univers itarios físicamente activos e inactivos

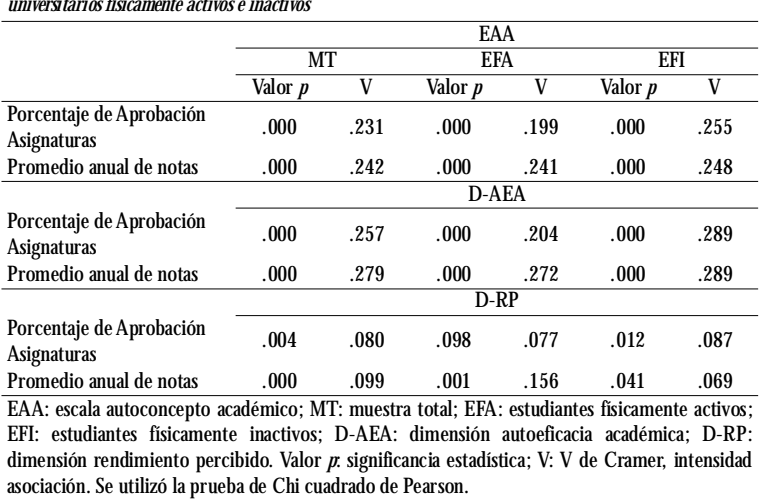

\section{Discusión}

Esta investigación tuvo como objetivo asociar el autoconcepto académico con el rendimiento académico en estudiantes universitarios chilenos físicamente activos e inactivos. Los principales resultados indican que existe correlación directa y asociación estadísticamente significativa entre las variables del estudio, demostrando que a mayor autoconcepto académico mejor es el rendimiento académico de los estudiantes que componen el total de la muestra, independientemente de la práctica de actividad física. Esta situación es concordante con investigaciones previas que indican asociación positiva entre el autoconcepto académico con el rendimiento académico de los estudiantes (Marsh \& Martin, 2011; Wang \& N eihart, 2015; K halaila, 2015; M éndez, 2016). No obstante, esta relación no muestra ser mejor en estudiantes físicamente activos, lo que difiere de lo manifestado en investigaciones que relacionan el autoconcepto académico con la práctica regular de actividad física (O netti-O netti, Chinchilla-M inguet, $M$ artins $\&$ Castillo-Rodriguez, 2019), así como aquellas que indican que la práctica de actividad física se asocia a un mejor rendimiento académico (Godoy-Cumillaf. et al., 2015; Iglesias, Planells \& M olina-López, 2019; RuizAriza, De laTorre-Cruz, Suárez-M anzano \& M artínezLópez, 2018; Ruiz-Hermosa, Alvarez-Bueno, CaveroRedondo, Martinez-Vizcaino, Redondo-Tébar \& Sánchez-López, 2019).

En cuanto la EAA y su relación con el rendimiento académico, es posible señalar que las pruebas de correlación y asociación arrojaron resultados estadísticamente significativos, evidenciando que ambas variables se encuentran relacionadas al analizar el total de la muestra. Los valores $r$ fueron positivos, lo que manifiesta una relación directa entre ambas variables, así, a mayor puntaje de laEAA, los estudiantes presentan mejor rendimiento académico, lo que es coincidente con investigaciones previas (Elis \& Loomis, 2002; Lane, Lane \& Kyprianou, 2004; Marsh \& Martin, 2011; Wang \& N eihart, 2015; Khalaila, 2015; Véliz-Burgos \& A podacaU rquijo, 2012; M éndez, 2016). Un asunto relevante radica en que el rendimiento académico se correlaciona con mayor fuerza estadística y se asocia con mayor intensidad a la EAA y sus dimensiones (AEA y RP) cuando se utiliza el promedio anual de notas, por sobre el porcentaje de aprobación de asignaturas, lo que podría significar que las notas son un indicador más certero para determinar el rendimiento académico, debido a que las notas, son el resultado de una serie de factores vinculados que aportan una base común de comparación respecto a una escala de notas oficializado (Rosas, 2011; Barahona, 2014).

En cuanto a la práctica de actividad física, el 36\% de 
los estudiantes evaluados declaró ser físicamente activo, situación concordante con la última encuesta nacional de hábitos de actividad física y deporte donde se informa que un $42.6 \%$ de los jóvenes chilenos situados entre los 18 y 29 años es físicamente activo (Ministerio del Deporte, 2018). Por su parte, si bien la EAA como sus dimensiones (AEA y RP) correlacionaron directa y positivamente con el rendimiento académico en el total de la muestra, al diferenciar a los estudiantes en físicamente activos y físicamente inactivos, no se puede sostener quelos primeros poseen un mejor autoconcepto académico y mejor rendimiento académico respecto a los segundos. De hecho, la relación entre la EAA y el rendimiento académico, comparando a los EFA y los $\mathrm{EFI}$, si bien manifiesta una correlación estadísticamente significativa para ambos grupos, los valores $r$ sistemáticamente evidencian una fuerza estadística mayor entre losEFI y el porcentaje de aprobación de asignaturas, mientras losEFA se relacionan con mayor fuerza estadística con el promedio anual de notas. Ello podría apoyar la idea expresada previamente respecto a que las notas pueden ser un indicador ideal para evaluar el rendimiento académico de los estudiantes $A$ su vez, es posible indicar que existe asociación estadísticamente significativa entre EAA y su dimensión AEA con el rendimiento académico tanto en los EFA como en los EFI, con una intensidad media según el estadístico $\mathrm{V}$ de Cramer, sin embargo, al considerar la dimensión RP, se presentan resultados menos esclarecedores, pues no siempre existe asociación entre los factores del rendimiento académico y los EFA y EFI y, en las ocasiones que se presenta asociación, el estadístico V de Cramer es cercano a cero, lo que corresponde a una asociación de muy baja intensidad.

Por lo tanto, los resultados de nuestro estudio difieren parcialmente con la evidencia científica que manifiesta un importante vínculo entre la práctica regular de actividad física con mejor desempeño académico (Godoy-Cumillaf. et a., 2015; Iglesias, Planells\& M olina López, 2019; Ruiz-Ariza, De la Torre-Cruz, SuárezManzano \& Martínez-López, 2018; Ruiz-Hermosa, Alvarez-Bueno, Cavero-Redondo, Martinez-Vizcaino, Redondo-Tébar \& Sánchez-López, 2019; O netti-O netti, Chinchilla-M inguet, Martins \& Castillo-Rodriguez, 2019), debido a que no se encontraron diferencias significativas en las correlaciones y asociaciones de la EAA y sus dimensiones con el rendimiento académico al analizar la muestra por separado entre EFA y EFI. Sin embargo, se encontraron correlaciones y asociaciones estadísticamente significativas en el total de la mues- tra, por lo que es posible afirmar que el autoconcepto académico se vincula positivamente con el rendimiento académico en estudiantes universitarios.

Entre las principales limitaciones del estudio se encuentran: a) la obtención de información por medio de encuestas en línea y auto reportadas, lo que podría sesgar las respuestas de los estudiantes; b) la diversidad de carreras a la que pertenecían los estudiantes que podría esconder las características y percepciones específicas de cada disciplina. Como principales fortalezas se encuentran: a) el tamaño de la muestra que corresponde al 23\% de los estudiantes matriculados en la universidad analizada; b) el uso de una encuesta validada en el contexto universitario chileno, lo que otorga informa ción más precisa del autoconcepto académico. En futuras investigaciones se podría vincular el autoconcepto académico y el rendimiento académico con otras características relevantes de los estudiantes, como su nivel socioeconómico, los antecedentes educativos de sus pa dres o tutores legales, las metodologías de enseñanza aprendizaje de los profesores, o las posibles distinciones por área del conocimiento o carreras a las que pertenecen los estudiantes.

\section{Conclusión}

A mayor autoconcepto académico mejor es el rendimiento académico en estudiantes universitarios, independientemente a la práctica de actividad física. En este sentido, sería relevante propiciar en las instituciones universitarias el desarrollo y fortalecimiento del autoconcepto académico de los estudiantes, mediante programas de apoyo y reforzamiento en base a la exploración de la imagen que perciben de su desempeño basal en diversas tareas y en la percepción de sus propias capacidades para la identificación, automonitoreo y resolución de problemas.

\section{Referencias}

Altbach, P., Reisberg, L., \& Rumbley, L. (2009). Trends in Global Higher Education: Tracking an Academic Revolution. Paris: UNESCO. Recuperado de https: / / s3. amazonaws.com/ academia.edu.documents/ $30910755 /$ A I t bach__ Reisberg_ R umbley_ T racking_an_Academic_Revolution__UNESCO_2009. $p d f$ ? $r$ e s p o n s e - conte nt disposition $=$ inline $\% 3$ B $\% 20$ fil ename $\% 3$ DTrends in_global higher_education_Tracki.pdf\&X-AmzAlgorithm $=$ =AW S4- ${ }^{-}$MAC AranedaGuirriman, C., Sallán,J. G., Pedraja Rejas, L., \& Rodríguez- 
Ponce, E. (2018). Percepciones sobre el perfil del estudiante universitario en el contexto delaeducacion superior demasas: Aproximaciones desde Chile. Interciencia, 43(12), 864-870. Recuperado dehttps:/ / www.interciencia.net/ wp-content/ uploads/2018/ 12/ 864-ARANEDA-43_12.pdf

Barahona, P. (2014). Factores determinantes dē rendimiento aca démico de losestudiantes delaU niversidad deAtacama. Estudios Pedagógicos, 40(1), 25-39. https:/ / doi.org/ 10.4067/ S0718-07052014000100002

Bernasconi, A. (2015). La educación superior de Chile:Transformación, desarrolloy crisis. Santiago, Chile.: EdicionesUC.

Biddle, S., \&Asare, M. (2011). Physical activity and mental health in children and adolescents: areview of reviews. British Journal of Sports M edicine, 45(11), 88-95. https:/ / doi.org/ 10.1136/ bjsports-2011-090185

Brunner, J., \& Ganga, F. (2016). Dinámicasdetransformación en la educación superior latinoamericana: Desafíos para la gobernanza. Opción Revista de Ciencias H umanas y Sociales, 32, 12-35. Recuperado de http:/ / www. redalyc. org/ pdf/ 310/ 31047691002.pdf

Cardenal, V., \& Fierro, A. (2003). Componentes y correlatos del autoconcepto en la escalade Piers- Harris. Estudios de Psicología., 24, 101-111. https:// doi.org/ 10.1174/ 021093903321329094

Carvajal, C. M., González, J. A. , \& Sarzoza, S. J. (2018). Variables Sociodemográicas y A cadémicas Explicativas delaD eserción deEstudiantesen laFacultad de CienciasN aturales de laU niversidad dePlayaAncha(Chile). Formación universitaria., 11(2), 3-12. https:/ / doi.org/ 10.4067/ S071850062018000200003

Cossio-Bolaños, M., Viveros-Flores, A., Castillo-Retamal, M., Vargas-Vitora, R., Gatica, P., \& Gómez-Campos, R. (2015). Patrones de actividad física en adolescentes en función del sexo, edad cronológica y biológica. Nutrición clínica y dietética hospitalaria., 35(2), 41-47. https:/ / doi.org/ 10.12873/ 352 cossio

Díaz, M .,A podaca U rquijo, P.,Arias, J., Escudero,T., Rodríguez, S., \&Vidal, J. (2002). Evaluación del RendimientoAcadémico en laEnseñanzaSuperior. Comparación deresultadosentrealumnosprocedentes de laLO GSE y del COU. Revista del nvestigación Educativa, 20(2), 357-383. Recuperado de https:/ / revistas.um.es/ rie/ article/ view/ 98971/ 94561

Donoso, S. \& Schiefelbein, E. (2007). Análisis de los modelos explicativos deretención deestudiantesen launiversidad: una visión desde la desigual dad social. Estudios pedagógicos, 33(1), 7-27. https:/ / doi.org/ 10.4067/ S071807052007000100001

Donoso, S., Donoso, G., \&Arias, Ó. (2010). Inicizivas de retención de estudiantes de educación superior. Calidad en la Educación, 33, 15-61. https: / / doi.org/ 10.31619/ caledu.n33.138

Elis, S. M., \& Loomis, R. J. (2002). Utilizing need for cognitionand perceived self efficacy to predict academic performance. Journal of Applied Social Psychology., 32, 1687-1702. https:/ / doi.org/ 10.1111/ j.1559-1816.2002.tb02770.x

Espinoza, O., \& González, L. E. (2016). Laeducación superior en
Chile y la compleja transición desde el régimen de autofinanciamiento haciael régimen degratuidad. Revista Latinoamericana de Educación Comparada, 7(10), 35-51. Recupera do de https:/ / dialnet. unirioja. es/ servlet/ articulo?codigo $=6562408$

Ferla, J., Valcke, M., \& Cai, Y. (2009). A cademic self-efficacy and academic self-concept: Reconsideringstructural relationships. Learning and Individual Differences, 19(4), 499-505. https:/ / doi.org/ https:/ / doi.org/ 10.1016/ j.lindif.2009.05.004

Ferreyra, M., Avitabile, C., Botero-Álvarez, J., Haimovich, F., \& U rzúa, S. (2017). Momento decisivo: la educación superior en Amé rica Latina y el Caribe. Washington, DC: Banco Mundial. https:/ / doi.org/ 10.1596/ 978-1-4648-1014-5

Garaigordobil, M ., \& Durá,A. (2006). Relacionesdel autoconcepto y la autoestima con la sociabilidad, estabilidad emocional y responsabilidad en adolescentes de 14 a 17 años. Revista Análisis y M odificación de Conducta., 32, 37-64. https:/ / doi.org/ 10.33776/ amc. v32i141.2132

Garbanzo, G. M. (2007). Factoresasociadosal rendimiento acadé mico en estudiantesuniversitarios, unareflexión desdelacalidad de laeducación superior pública. Revisa Educación, 31(1), 43-63. Recuperado dehttps. / www. redalyc. org/ pdf/ 440/ 44031103.pdf

Garber, C. B. (2011).American CollegeofSportsM edicineposition stand. Quantity and quality of exercise for developing and maintaining cardiorespiratory, musculoskeletal, and neuromotor fitnessin apparently healthy adults: guidance for prescribing exercise. Medicine and science in sports and exercise, 43(7), 1334-1359. https:// doi. org/ 10.1249/ MSS.0b013e318213fefb

Gazzola, A. L., \& Didriksson, A. (2008). Tendenciasdela Educación Superior en América Latina y el Caribe. Caracas: IESALCUNESCO.

Gedda, R. (2016). Construcción comprensiva de dimensiones paraunaevaluación diagnósticadelapermanenciauniversitaria en estudiantes primerageneración. Un estudio de caso. Revista Investigaciones en Educación, 16(2), 121-140. Recuperado de http:/ / revistas, ufro.cl/ ojs/ index. php/ educacion/ article/ view/ 1130

Godoy-Cumillaf, A., Valdés-Badilla, P., Fariña-Herrera, C., Cárcamo-Mora, Medina Herrera, B., Meneses-Sandoval, E., . . . Durán-Agüero, S. (2015). Asociación entrelacondición física, estado nutricional y rendimiento académico en estudiantesde educación física. Nutrición Hospitalaria, 32(4), 1722-1728. https:/ / doi.org/ 10.3305/ nh.2015.32.4.9592

Grissom, J. (2005). Physical Fitness and Academic Achievement. JEPonline, 8(1), 11-25. Recuperado de https:// peaceful playgrounds.com/ pdf/ right-to-recess/ fitnessacademic-achievement. pdf

Hattie, J. (1992). Self-concept. NJ, Erlbaum: Hillsdale.

Iglesias, A., Planells, E., \& M olina-López, J. (2019). Prevalenciade sobrepeso y obesidad, hábitos al imentarios y actividad físicay su relación sobre el rendimiento académico (Prevalence of overweight and obesity, exercise, and dietary habits, and their relation withacademicachievement). Retos, 36(36), 167-173. 
https:/ / doi.org/ 10.47197/ retos.v36i36.66873

Khalaila, R. (2015). The relationship between academic selfconcept, intrinsic motivation, test anxiety, and academic achievement among nursing students: Mediating and moderating effects N urse Education Today, 35(3), 432-438. https: / / doi.org/ 10.1016/ j.nedt. 2014.11.001

Lane, J., Lane, A. M., \& Kyprianou, A. (2004). Self-efficacy, selfesteem and their impact on academic performance. Social Behavior and Personality:An international journal, 32 (3), 247-256. https: / doi.org/ 10.2224/ sbp. 2004.32.3.247

Ltorre, A., Arnal, J., \& Del Rincón, D. (2003) Bases metodológicas dela investigación educativa. Barcelona: Experiencia.

Marsh, H.W., \& Craven, R. G. (1997). Academic Seff-concept:Beyond thedustbowl.. (a. a. G. Phye. Handbook of classroom assessment: Learning, Ed.) O rlando, FL.: Academic Press.

Marsh, H.W., \& Hau, K.T. (2003). Big-Fish- Little-Pond effect on academic self-concept: A cross-cultural (26-country) test of the negative effects of academically selective schools. American Psychologit, 58(5), 364-376. https:/ / doi.org/ https:/ / doi.org/ 10.1037/ 0003-066X.58.5.364

Marsh, H. W., \& Shavelson, R. (1985). Self-Concept: Its Multifaceted, Hierarchical Structure. Educational Psychologit, 20(3), 107-123. https:// doi.org/ doi:10.1207/ s15326985ep2003_1

Marsh, H., \& Martin, $\bar{A}$. (2011). Academic self concept and academic achievement: Relations and causal ordering British Journal of Educational Psychology,81(1), 59-77. https:/ / doi.org/ 10.1348/000709910X503501

Méndez, J. (2016).AutoconceptoA cadémico y RendimientoAca démico en Estudiantes de laU niversidad de LaFrontera. Aná lisis Comparativo por Facultad. Revista Investigacionesen Educación, 16(1), 169-188. Recuperado de http:/ / revistas.ufro.cl/ ojs/ index. php/ educacion/ article/ view/ 1119

M éndez, J., \& Gálvez, J. (2018). Propiedades psicométricas de la Escala de Autoconcepto Académico (EAA) en estudiantes universitarios chilenos. Liberabit, 24(1), 131-145. https:/ / doi.org/ 10.24265/ liberabit.2018.v24n1.09

Ministerio del Deporte. (2018). Encuesta Nacional de Hábitos de Actividad Física y Deporte 2018 en Población de 18 años y más Santiago. Recuperado de http:// www. mindep.cl/ wpcontent/ uploads/ 2019/01/ Encuesta-Act.F\%C3\%ADsicay-Deportes-2018-VF.pdf

Onetti-O netti,W., Chinchilla-M inguet, J., M artins, F., \& CastilloRodriguez, A. (2019). Self-Concept and Physical Activity: Differences Between High School and U niversity Students in Spain and Portugal. Frontiers in Psychology, 10, 1333. https:/ / doi.org/ 10.3389/ fpsyg 2019.01333

Rodríguez, S., Fita, E., \&Torrado, M. (2004). El rendimiento aca démico en latransición secundaria-universidad. Revista deEducación, 334(1), 391-414. Recuperado de http:// www.revistaeducacion.educacion.es/ re334/ re334_22.pdf

Rosas, R. (2011). Éxito Académico Universitario: Problemas de su definición, medición y predicción. Phsyke, 1(1), 25-39. Re cuperado de http:/ / www.psykhe.cl/ index. php/ psykhe/ article/ view/ 14/ 14
Rudolph, D. \& M cAuley, E. (1995). Physical activity, aging, and psychological well-being J Aging Phys Act, 3, 67-96. https:/ / doi.org/ 10.1123 / japa 3.1.67

Ruiz-Ariza, A., De laTorre-Cruz, M. J., Suárez-Manzano, S., \& Martínez-López, E.J. (2018). A poyo haciala actividad físicay rendimiento académico independientemente del estatus socioeducativo parental (Supporttowardsphysical activity and academic performanceregardl essof parental socio-educational status). Retos, (35), 208-212. https./ / doi.org/ 10.47197/ retos. v0i35. 60545

Ruiz-Hermosa, A., Alvarez-Bueno, C., Cavero-Redondo, I., Martinez-Vizcaino,V., Redondo-Tébar, A. \& Sánchez-López. (2019). Active Commuting to and from School, Cognitive Performance, and Academic Achievement in Children and Adolescents: A Systematic Review and MetaAnalysis of O bservational Studies International Journal of Environmental Rescarch and Public H ealth., 16, 1839-1861. https: / / doi.org/ 10.3390/ ijerph16101839

Santana, C., Azevedo, L., Cattuzzo, M., Hill, J., \&Andrade, L., \&. (2017). Physical fitness and academic performance in youth: A systematic review. Medicine \& Science in Sports, 27(6), 579603. https:/ / doi.org/ 10.1111/ sms. 12773

Schmidt, V., Messoulam, N., \& Molina, F. (2008). Autoconcepto académico en adolescentes de escuelas medias: presentación deun instrumento parasu evaluación. Revista Iberoamericana de Diagnósico e Avaliação Psicológica, 1(25), 81-106. Recuperado de http:/ / www. redalyc.org/ pdf/ 4596/ 459645445005.pdf

Shavelson, R., Hubner, J., \& Stanton, G. (1976). Self-Concept: Validation of Construct Interpretations. Review of Educational Research, 46(3), 407-441. https: / / doi. org/ https: / / doi.org/ 10.3102/00346543046003407

Teixeira, P., Sunwoong, K., Landoni, P., \& Zulfiqar, G. (2017). Rethinking thePublic-PrivateM ixin Higher Education. Rotterdam, Holanda: Sense.

Vélez, A., \& Roa, C. (2005). Factors associated with academic performance in medical students. Revista Educación Médica, 8(2), 24-32. Recuperado dehttp:/ / scielo. isciii.es/ pdf/ edu/ v8n2/ original1. pdf

Véliz-Burgos, A., \& A podacaU rquijo, P. (2012). Niveles de autoconcepto, autoeficaciaacadémicay bienestar psicológico en estudiantes universitarios de la ciudad deTemuco. Salud \& Sociedad, 3(2), 1313-150. Recuperado de http:// pepsic. bvsalud. org/ pdf/ salsoc/ v3n2/ v3n2a02.pdf

Véliz-Burgos, A., Dörner, A., \& Sandoval, S. (2016). Relación entre autoconcepto, autoeficacia académica y rendimiento aca démico en estudiantes de salud de Puerto Montt, Chile. EDUCADI, 1(1), 97-109. https:/ / doi.org/ 10.7770/ EDUCADI-V1N 1-ART 1003

Wang, C., \& Neihart, M. (2015). Academic Self-Concept and Academic Self-Efficacy: Self-Beliefs Enable Academic Achievement of Twice-Exceptional Students. Journal Roeper Review, 37(2), 63-73. https:// doi.org/ 10.1080/ 02783193.2015 .1008660 